\title{
Prediction of intrinsic ferroelectricity and large piezoelectricity in monolayer arsenic chalcogenides
}

\author{
Weiwei Gao*,† and James R. Chelikowsky,,,,$++ \uparrow$ \\ $\dagger$ Center for Computational Materials, Oden Institute for Computational Engineering and \\ Sciences, The University of Texas at Austin, Austin, TX 78712 \\ $\ddagger$ Department of Physics, The University of Texas at Austin, Austin, TX 78712 \\ \McKetta Department of Chemical Engineering, The University of Texas at Austin, \\ Austin, TX 78712 \\ E-mail: weiwei@ices.utexas.edu; jrc@utexas.edu
}

\begin{abstract}
Two-dimensional materials that exhibit spontaneous electric polarization are of notable interest for functional materials. However, despite many two-dimensional polar materials are predicted in theory, the number of experimentally confirmed two-dimensional ferroelectrics are still far less than bulk ferroelectrics. We provide strong evidence that the Pmn $2_{1}$ phase of arsenic chalcogenides $\mathrm{As}_{2} \mathrm{X}_{3}(\mathrm{X}=\mathrm{S}$, Se, and $\mathrm{Te}$ ), which include the recently isolated monolayer orpiment, are intrinsic ferroelectrics and demonstrate strong in-plane piezoelectricity. We found the calculated energy barriers for collectively reversing the electric polarization or moving a $180^{\circ}$ domain wall are reasonable compared to previously reported ferroelectrics. We propose a high-symmetry structure (with Pmmn space group) transforms into the ferroelectric Pmn2 $2_{1}$ phase by a soft $\mathrm{B}_{2 u}$ phonon mode. By studying other soft modes of the high-symmetry Pmmn structure, we identify several undiscovered metastable polymorphs, including a polar phase (with a $\mathrm{P} 22_{1}$ space group) with sizable piezoelectricity.
\end{abstract}

\section{Keywords}

ferroelectricity; piezoelectricity; two-dimension material; first-principles calculations; polymorphism

\section{Introduction}

Materials lacking inversion symmetry may display useful properties such as piezoelectricity and ferroelectricity, which have wide applications in modern industries. In particular, ferroelectric materials are not only prototypical systems for studying spontaneous symmetry breaking and structural phase transitions, but also key components for non-volatile memory devices, piezoelectric sensors, photocatalysis, and many other technologically important applications. ${ }^{1-3}$ Driven by the need for further miniaturization of electronic devices, researchers have devoted significant efforts to reduce the thickness of thin-films ferroelectrics. ${ }^{4-6}$ Despite the depolarization field, ${ }^{7-9}$ which usually inhibits the electric polarization of thin-film ferroelectrics, a few groups have demonstrated ferroelectricity sustains in bulk ferroelectrics with thickness down to $\sim 1 \mathrm{~nm} .{ }^{10,11}$ The recent discovery of ferroelectricity in monolayer or fewlayer Van der Waals (vdW) materials offer new 
opportunities for shrinking the size of ferroelectric devices to the atomically thin regime. ${ }^{12-16}$ Compared to conventional bulk ferroelectrics, a key advantage of two-dimension vdW materials is free of dangling bonds on the surface. First-principles calculations also show that a large number of two-dimensional (2D) materials are piezoelectric. ${ }^{17-19}$ Remarkably, some of them ${ }^{17,20}$ even demonstrate giant piezoelectric effects, which can be more than two-orders-ofmagnitude stronger than bulk piezoelectric materials.

Currently, our understanding of the fundamental physical properties of $2 \mathrm{D}$ piezoelectric and ferroelectric systems is in an early stage, and the lack of a robust and economical fabrication process for high-quality 2D ferroelectric samples hinders mass production and applications. ${ }^{21}$ Among 2D ferroelectric materials predicted with first-principles theories, ${ }^{20,22-27}$ only a few, such as monolayer $\mathrm{SnS},{ }^{28} \mathrm{SnSe},{ }^{29} \mathrm{SnTe}^{14}$ and $\mathrm{In}_{2} \mathrm{Se}_{3},{ }^{15}$ have so far been synthesized and confirmed to be ferroelectric. First-principles prediction of piezoelectricity or switchable electric polarization in readily fabricated $2 \mathrm{D}$ materials is important for enriching the toolbox of 2D non-centrosymmetric materials with technological interests.

Through first-principles calculations, we show ample evidence that three monolayer arsenic chalcogenides $\left(\mathrm{As}_{2} \mathrm{X}_{3}\right)$ with the $\mathrm{Pmn} 2_{1}$ space group will exhibit spontaneous and reversible in-plane polarization. Among these three materials, the $\mathrm{Pmn}_{1} \mathrm{As}_{2} \mathrm{~S}_{3}$, i.e., monolayer orpiment, has recently been isolated through mechanical exfoliation. ${ }^{30}$ Moreover, we predict the existence of several novel metastable polymorphs of $\mathrm{As}_{2} \mathrm{~S}_{3}$. Both ferroelectric monolayer orpiment and these new polymorphs can be related to the soft zone-center modes of a hypothetical high-symmetry phase. Remarkably, our calculations show some of these polymorphs have large piezoelectric coefficients comparable to those of group IV-VI compounds. ${ }^{20}$

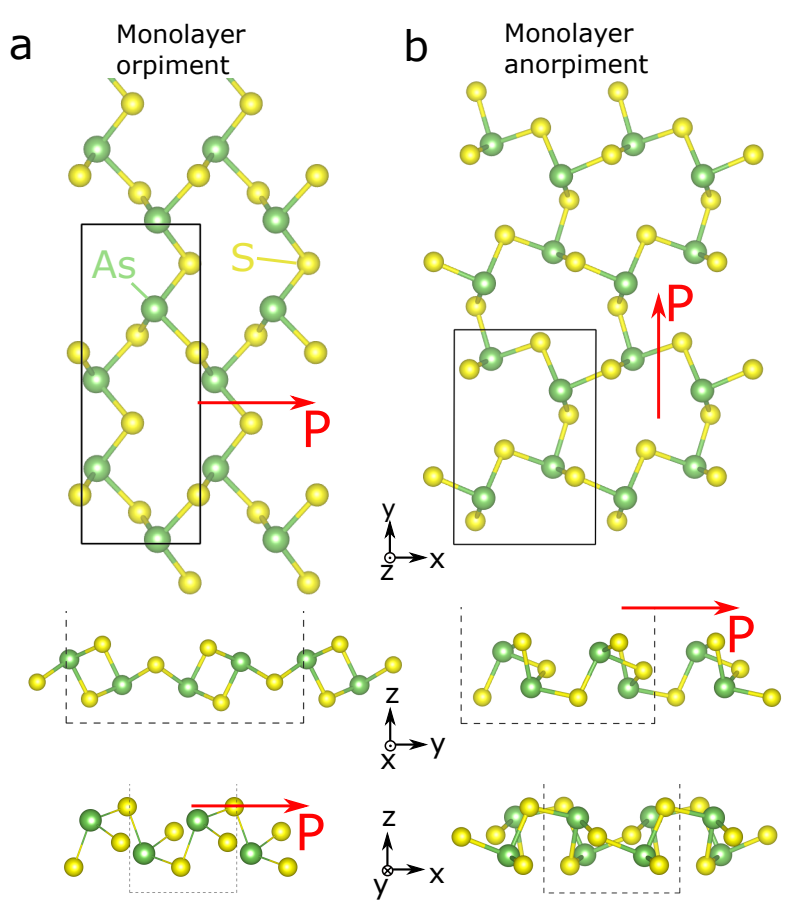

Figure 1: The crystal structures of (a) monolayer orpiment and (b) monolayer anorpiment, plot with VESTA. ${ }^{31}$ The red arrows show the direction of polarization.

\section{Results and Discussion}

Under ambient conditions, bulk $\mathrm{As}_{2} \mathrm{~S}_{3}$ can be either amorphous or crystalline. Bulk orpiment and anorpiment, which were found in natural minerals, ${ }^{32}$ are two common crystalline $\mathrm{As}_{2} \mathrm{~S}_{3}$ phases with noncentrosymmetric layered structures bounded by vdW interactions. To date, two-dimensional anorpiment has not been synthesized, while monolayer and fewlayer orpiment have been successfully exfoliated and demonstrates better chemical stability than phosphorene under low light conditions. ${ }^{30}$ Our calculated total energy of monolayer orpiment is lower than that of monolayer anorpiment by $73 \mathrm{meV} /$ formula unit (f.u.), suggesting better stability of monolayer orpiment compared to monolayer anorpiment. A finite bandgap is required for sustaining the ferroelectricity of 2D materials with in-plane polarization. Monolayer orpiment and anorpiment have indirect bandgaps around $2.2 \mathrm{eV}$ calculated with the Perdew-Burke-Ernzerhof (PBE) functional. ${ }^{33}$ The band structures are presented in the Supporting Information. 


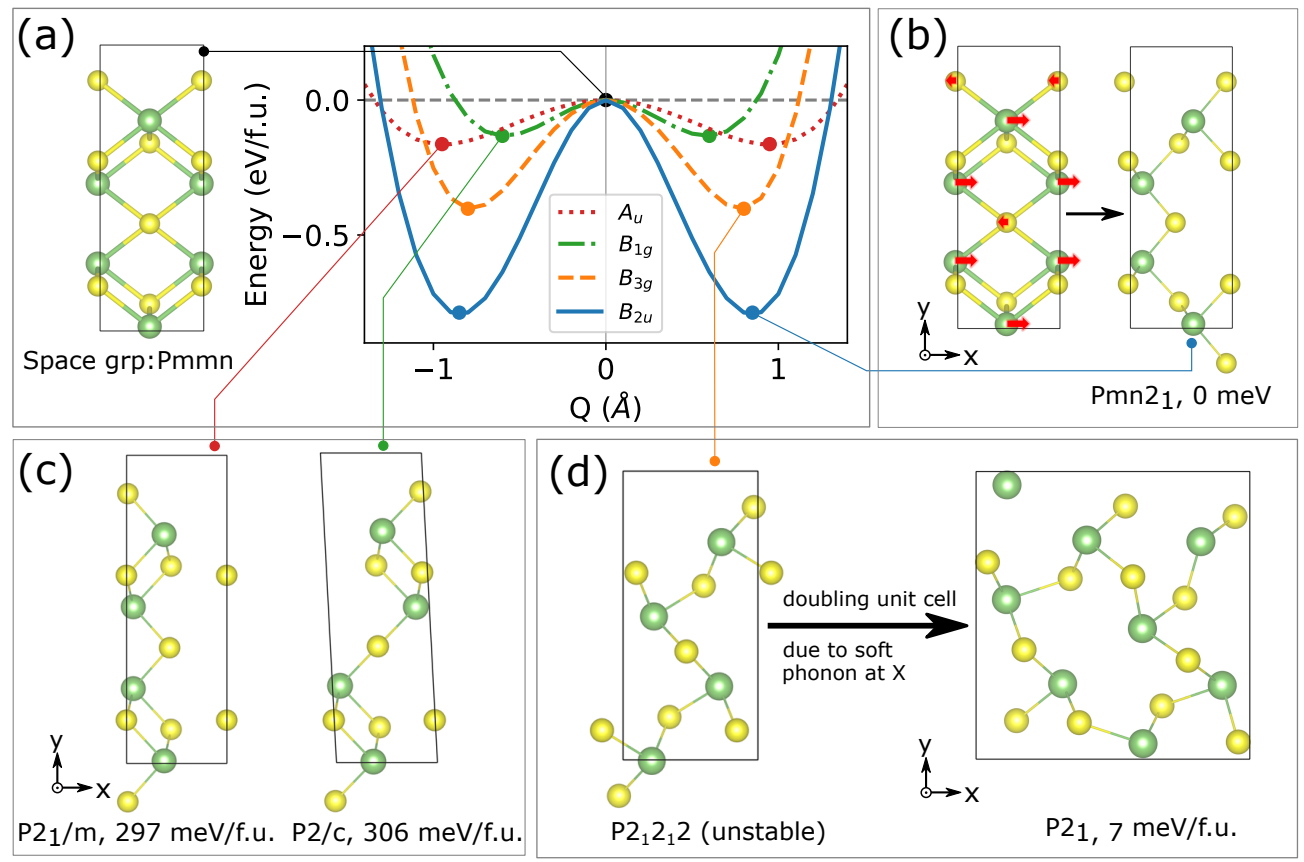

Figure 2: (a) (left) The high-symmetry structure with space group Pmmn and (right) the change of total energy under collective atomic displacements of the Pmmn structure with frozen-in soft phonon mode $\mathrm{A}_{u}, \mathrm{~B}_{1 g}, \mathrm{~B}_{3 g}$, and $\mathrm{B}_{2 u}$. The displacement vector $\Delta \mathbf{R}_{p}$ is proportional to the polarization vector of phonon $p: \Delta \mathbf{R}_{p}=Q \cdot \mathbf{u}_{p}$. (b) Schematic plot of the transform from Pmmn structure to monolayer orpiment through the $\mathrm{B}_{2 u}$ soft mode. The red arrows (not to scale) show the moving direction of corresponding atoms. (c) Unit cell of the metastable $\mathrm{P} 2{ }_{1} / \mathrm{m}$ phase and $\mathrm{P} 2 / \mathrm{c}$ phase. (d) Schematic plot of the stabilization of the $\mathrm{P} 2_{1} 2_{1} 2$ phase by doubling the unit cell due to soft modes at $X$ point.

The crystal structures of monolayer arsenic chalcogenides do not resemble those of other well-known 2D materials. As shown in Fig. 1(a), monolayer orpiment is highly anisotropic and consists of rings connected by six corner-sharing $\mathrm{AsS}_{3}$ units, which have a pyramidal shape. Monolayer orpiment has Pmn2 1 symmetry, which includes a mirrorreflection to the $x z$-plane, but no symmetry with the yz-plane, as illustrated in Fig. 1(a). Such symmetry properties allow a spontaneous electric polarization along the $x$-axis. In comparison, monolayer anorpiment has a more irregular structure and electric polarization pointing in the $y$-direction, as illustrated in Fig. 1(b).

Bulk $\mathrm{As}_{2} \mathrm{Se}_{3}$ can be found in mineral laphamite with a similar structure as orpiment, ${ }^{34}$ while bulk $\mathrm{As}_{2} \mathrm{Te}_{3}$ with the orpimentlike structure is yet to be found. Our calculations show monolayer $\mathrm{As}_{2} \mathrm{Se}_{3}$ and $\mathrm{As}_{2} \mathrm{Te}_{3}$ with the orpiment-like structure (Pmn2 $2_{1}$ symme- try) are dynamically stable, while those with the anorpiment-like structure (Pc space group) demonstrate dynamical instability with imaginary phonon modes.

Using first-principles methods based on modern polarization theory, ${ }^{35,36}$ we calculate that monolayer orpiment has a spontaneous electric polarization of $71 \mathrm{pC} / \mathrm{m}$. According to the experimental structure of bulk orpiment, ${ }^{32,37}$ the electric polarization in neighboring layers aligns in an antiferroelectric order. Therefore, bulk orpiment shows no macroscopic polarization and the net polarization of a few-layer orpiment shows an odd-even effect. Only samples with odd numbers of layers show net electric polarization.

As a classical example of displacive transitions, the ferroelectric phase transition of perovskite oxide like $\mathrm{PbTiO}_{3}$ is explained by a zone-center vibrational mode which vanishes at the phase transition. Similarly, we propose the ferroelectricity of monolayer orpiment is also 

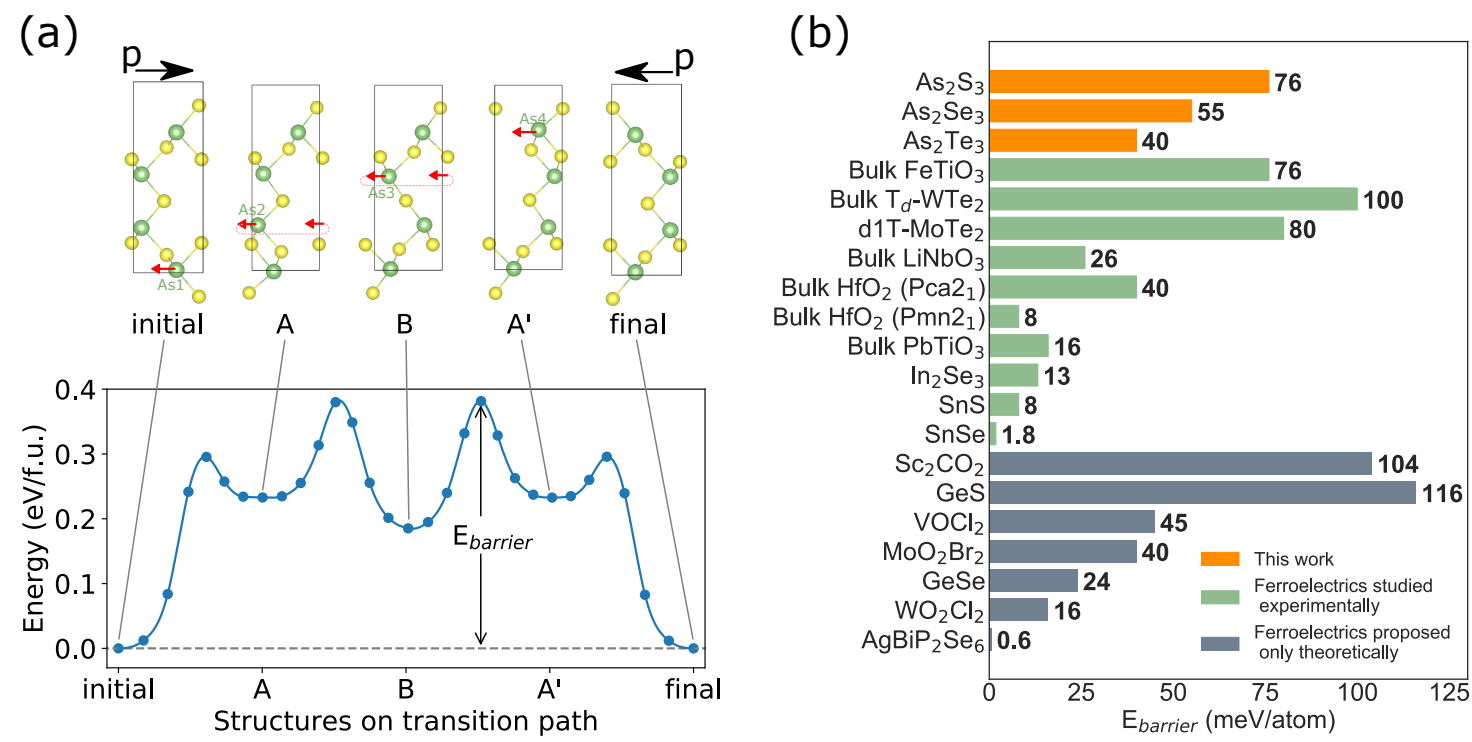

Figure 3: (a). (top) Selected intermediate states on the transition path of inverting the polarization direction of monolayer orpiment. Red arrows show the main movement of As ions between consecutive intermediate structures. The dashed curves are used to represent the atomic displacements that cross the unit-cell boundary. (bottom) The evolution of the total energies of intermediate structures along the transition path. (b). A comparison between the theoretical energy barriers $E_{\text {barrier }}$ of the polarization-reversing process of arsenic chalcogenide and other ferroelectrics. The values from previous work are all calculated with density functional theory.

driven by a soft mode of a high-symmetry structure with space group Pmmn. The unit cell of the Pmmn structure is shown schematically in Fig. 2(a). Different from monolayer orpiment which only has mirror symmetry to $x z$ plane, the Pmmn structure of $\mathrm{As}_{2} \mathrm{~S}_{3}$ has additional mirror symmetry with $y z$-plane. This high-symmetry Pmmn structure is dynamically unstable with five soft optical phonon modes at the $\Gamma$ point. To quantify contributions of a zone-center soft phonon mode to the structural transition from the high-symmetry Pmmn structure to Pmn2 1 phase, we calculate the projection of the atomic displacement vector $\Delta \mathbf{R}=\mathbf{R}_{P m m n}-\mathbf{R}_{P m n 2_{1}}$ on soft phonon modes:

$$
\eta[p]=\frac{\Delta \mathbf{R}}{|\Delta \mathbf{R}|} \cdot \mathbf{u}_{p}
$$

where $\mathbf{u}_{p}$ is the normalized polarization vector of a zone-center phonon $p$. We find $\eta\left[B_{2 u}\right]=$ $86 \%$, and other four soft zone-center modes contribute less than 1 percent to $\Delta \mathbf{R}$. This is expected since the $B_{2 u}$ mode is the only one that breaks the inversion symmetry and also has the deepest double-well potential curve among all zone-center soft modes. Therefore the $\mathrm{B}_{2 u}$ mode is the dominant phonon mode driving the structural transition from the unstable Pmmn structure to the $\mathrm{Pmn}_{1}$ phase of $\mathrm{As}_{2} \mathrm{~S}_{3}$. As shown in Fig. 2(b), the main effect of $\mathrm{B}_{2 u}$ optical mode is to shift the As atoms along the $x$-axis and break the reflection symmetry to $y z$ plane. The relative shifts between different $\mathrm{S}$ atoms are smaller comparing to the displacement of As atoms in the $\mathrm{B}_{2 u}$ mode.

We also examine the roles of other four soft zone-center modes, namely $\mathrm{B}_{3 g}, \mathrm{~B}_{1 g}, \mathrm{~B}_{3 u}$, and $\mathrm{A}_{u}$ modes, by collectively displacing atomic coordinates of the high-symmetry Pmmn structure by $\Delta \mathbf{R}_{p}=Q \cdot \mathbf{u}_{p}$, where $\mathbf{u}_{p}$ is the normalized polarization vector of phonon mode $p$. As shown in Fig. 2(a), one can easily identify structures that correspond to the local minima (shown as small spheres) on the double-well curve of total energy versus general coordinate $Q$. Further relaxing local minimal structures may lead to new metastable phases of $\mathrm{As}_{2} \mathrm{~S}_{3}$. Since the structural relaxation moves the local minima that correspond to the $\mathrm{B}_{3 u}$ mode back 
to monolayer orpiment, we will not discuss it further.

Interestingly, $\mathrm{B}_{3 g}$ and $\mathrm{B}_{1 g}$ modes transform the high-symmetry Pmmn structure into metastable $\mathrm{P} 2 / \mathrm{c}$ and $\mathrm{P} 2 / 1 \mathrm{~m}$ phases, respectively. As shown in Fig. 2(c), both $\mathrm{P} 2 / \mathrm{c}$ and $\mathrm{P} 22_{1} / \mathrm{m}$ phase show unusual one-dimensional chain structures consist of interconnected $\mathrm{AsS}_{3}$ pyramidal units. These two phases show zero macroscopic polarization since the dipole moments of neighboring $\mathrm{AsS}_{3}$ pyramidal units point in opposite directions and thus cancel with each other.

A more complicated case is the soft $\mathrm{A}_{u}$ mode. Relaxing the local minima structure corresponding to the $\mathrm{A}_{u}$ mode leads to a dynamically unstable $\mathrm{P} 2{ }_{1} 2_{1} 2$ structure without a net electric polarization. Such an unstable structure has doubly-degenerate soft phonon modes at the $X$ point which can stabilize the structure by doubling the unit cell along $x$-axis. The final stable structure we find has the $\mathrm{P} 2_{1}$ space group symmetry and a rectangle unit cell with 20 atoms, as shown in Fig. 2(d). The P2 1 phase has a noncentrosymmetric structure with spontaneous polarization of $20 \mathrm{pC} / \mathrm{m}$ pointing in the $y$-direction. We confirmed the stability of $\mathrm{P} 2 / \mathrm{c}$, $\mathrm{P} 2{ }_{1} / \mathrm{m}$, and $\mathrm{P} 2{ }_{1}$ phases from their phonon spectra calculated with density functional perturbation theory ${ }^{38}$ and finite-temperature molecular dynamics ${ }^{39}$ trajectories. These results are presented in Supporting Information.

The total energy of the $\mathrm{P} 2{ }_{1}$ phase $\mathrm{As}_{2} \mathrm{~S}_{3}$ is 65 $\mathrm{meV} /$ f.u. lower than that of monolayer anorpiment, and only $7 \mathrm{meV} /$ f.u. higher than that of monolayer orpiment. Even though the P2/c and $\mathrm{P} 2_{1} / \mathrm{m}$ phases are shown to be metastable, they have total energies which are about 300 $\mathrm{meV} /$ f.u. higher than that of monolayer orpiment, because they are composed with onedimensional chain-like structures bounded by weak vdW forces. We mention only the zonecenter soft modes of Pmmn structures are studied in this work. It is also interesting to study the finite-momentum soft modes, which also appear in the phonon spectrum of the Pmmn structure and may lead to other interesting polymorphs.

We perform similar analyses on the soft modes of the high-symmetry Pmmn structure of $\mathrm{As}_{2} \mathrm{Se}_{3}$ and $\mathrm{As}_{2} \mathrm{Te}_{3}$. Like $\mathrm{As}_{2} \mathrm{~S}_{3}$, they both have a $\mathrm{B}_{2 u}$ mode driving the displacive transition to the corresponding $\mathrm{Pmn} 2_{1}$ phase. $\mathrm{As}_{2} \mathrm{Se}_{3}$ also has a metastable phase with $\mathrm{P} 2{ }_{1}$ space group. In Table 1, we list the space groups and the electric polarization of all stable polymorphs studied in this work. We find $P$ of Pmn2 1 phases decreases as the chalcogen element changes from sulfur to tellurium. A similar trend also appears in IV-VI monolayers. ${ }^{20}$ We explain this qualitatively with two arguments. First, The electrical polarization $\mathrm{P}$ is positively correlated to the difference between the electron negativity of $\mathrm{As}$ and the chalcogen elements. As the chalcogen element changes from $\mathrm{S}$ to Te, the reduced electronegativity results in a diminished polarization. Second, since the distortion amplitude $\left|Q_{\text {min }}\right|$ at the minima of the double-well potential of $\mathrm{B}_{2 u}$ mode decreases as the chalcogen element changes from $\mathrm{S}$ to $\mathrm{Te}$, the dipole moment, which is proportional to $\left|Q_{\min }\right|$, also decreases.

Table 1: Summary of the space group and electric polarization $P$ of different arsenic chalcogenides phases.

\begin{tabular}{ccc}
\hline Formula & Space group & $\begin{array}{c}P(\mathrm{pC} / \mathrm{m}) \\
\text { (The direction of } \mathrm{P})\end{array}$ \\
& $\begin{array}{c}\text { Pmn2 } \\
\text { (Monolayer } \\
\text { orpiment) }\end{array}$ & $71(\mathrm{x})$ \\
$\mathrm{As}_{2} \mathrm{~S}_{3}$ & $\mathrm{Pc}$ & \\
& (Monolayer & $47(\mathrm{y})$ \\
& anorpiment) & 0 \\
& $\mathrm{P} 2 / \mathrm{c}$ & 0 \\
& $\mathrm{P} 2_{1} / \mathrm{m}$ & $20(\mathrm{y})$ \\
\hline $\mathrm{As}_{2} \mathrm{Se}_{3}$ & $\mathrm{P} 2_{1}$ & $54(\mathrm{x})$ \\
& $\mathrm{Pmn} 2_{1}$ & $18(\mathrm{y})$ \\
\hline $\mathrm{As}_{2} \mathrm{Te}_{3}$ & $\mathrm{Pmn} 2_{1}$ & $45(\mathrm{x})$ \\
\hline
\end{tabular}

The reversibility of electric polarization is a necessary condition for ferroelectrics and also important for the application in data storage. Using the nudged-elastic-band method, ${ }^{40}$ we find a minimal-energy-barrier transition path 
for reversing the electric polarization of an infinite large monolayer orpiment. We show important intermediate structures and the corresponding energies on the transition path of $\mathrm{As}_{2} \mathrm{~S}_{3}$ in Fig. 3 (a). In the polarizationreversing process, three important intermediate structures labeled as $A, B$, and $A^{\prime}$ are found. $A$ and $A^{\prime}$ are related by a $180^{\circ}$ rotation around the $z$-axis. $B$ is structurally akin to the unstable $\mathrm{P} 2{ }_{1} 2_{1} 2$ structure. The initial and final structures on the transition path correspond to monolayer orpiment with electric polarization pointing in opposite directions. The process of reversing electric polarization goes in the sequence $\left\{\right.$ initial $\rightarrow A \rightarrow B \rightarrow A^{\prime} \rightarrow$ final $\}$, which consists of four major steps. Each step mainly involves shifting a single As atom along the $x$-axis. For example, in the first step $\{$ initial $\rightarrow A\}$, the major structural change is the displacement of As1 (i.e. the arsenic atom at the bottom of the unit cell shown in Fig. 3 (a)) along the negative $x$-direction. Similarly, in the second step $\{A \rightarrow B\}$, we observe the movement of As2 along the negative $x$-direction to pass the boundary of the unit cell. We emphasize that the switching process we presented here may not correspond to the global minimum barrier, since the changes of lattice vectors are not considered in our nudged-elasticband calculations and the electric dipoles can never be switched simultaneously in real situations. Previous work also shows that including the variation of lattice vectors can further lower the energy barrier. ${ }^{23,41}$ Nevertheless, our theoretical energy barrier $\mathrm{E}_{\text {barrier }}$ provides an upper bound for the activation energy of the real polarization-reversing process. With the similar approach, the energy profiles for reversing the electric polarization of $\mathrm{As}_{2} \mathrm{Se}_{3}$ and $\mathrm{As}_{2} \mathrm{Te}_{3}$ are calculated and shown in the Supporting Information.

In Fig. 3 (b), we compare calculated energy barriers $\mathrm{E}_{\text {barrier }}$ of $\mathrm{As}_{2} \mathrm{X}_{3}$ with those of other ferroelectrics, which are either studied experimentally ${ }^{15,16,25,28,42-49}$ or solely predicted in theory. ${ }^{26,27,45,50-52}$ Except $\mathrm{FeTiO}_{3}, \mathrm{LiNbO}_{3}$, $\mathrm{AgBiP}_{2} \mathrm{Se}_{6}$, and $\mathrm{PbTiO}_{3}$, the $\mathrm{E}_{\text {barrier }}$ in Fig. 3 are calculated with the nudged-elastic-band method. The range of calculated $E_{\text {barrier }}$ cov- ers two orders of magnitudes from $0.6 \mathrm{meV}$ to $116 \mathrm{meV}$. The $E_{\text {barrier }}$ of $\mathrm{As}_{2} \mathrm{X}_{3}$ are notably smaller than those of two room-temperature ferroelectrics, namely $T_{d}-\mathrm{WTe}_{2}{ }^{16}$ and monolayer $\mathrm{d} 1 \mathrm{~T}-\mathrm{MoTe}_{2},{ }^{42}$ and a few predicted ferroelectrics such as $\mathrm{GeS}^{45}$ and $\mathrm{Sc}_{2} \mathrm{CO}_{2},{ }^{23}$ but much larger than those of $\mathrm{CuInP}_{2} \mathrm{~S}_{6},{ }^{49} \mathrm{In}_{2} \mathrm{Se}_{3},{ }^{25}$ $\mathrm{SnS}^{45}$ and so on. Such comparisons suggest that the energy barriers of switching the polarization direction of $\mathrm{Pmn} 2_{1} \mathrm{As}_{2} \mathrm{X}_{3}$ are within a proper range.

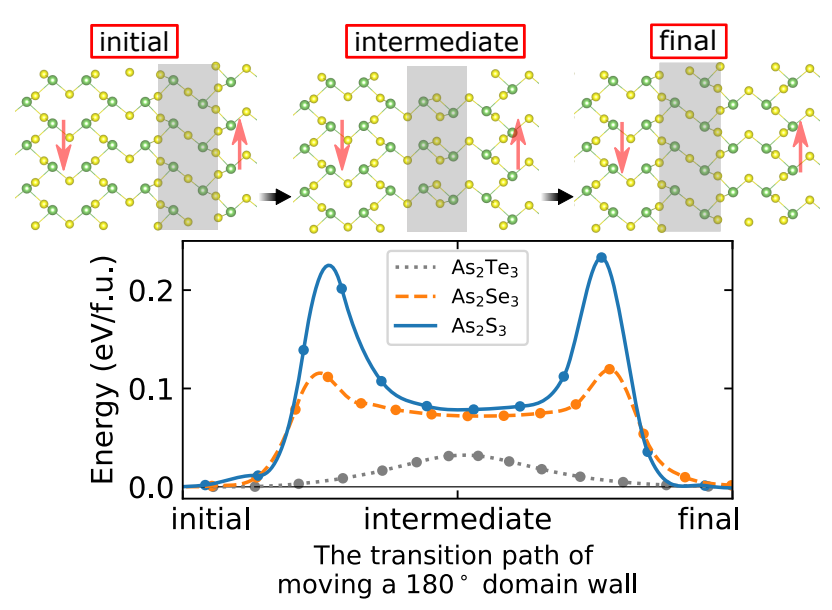

Figure 4: (top panel) Selected structures on the process of moving the $180^{\circ}$ domain wall in $\mathrm{As}_{2} \mathrm{~S}_{3}$. The shaded regions highlight the domain boundaries. (bottom panel) Total energies on the process of moving the domain walls of $\mathrm{As}_{2} \mathrm{~S}_{3}, \mathrm{As}_{2} \mathrm{Se}_{3}$, and $\mathrm{As}_{2} \mathrm{Te}_{3}$.

In practical situations, domain-wall shifting and domain growing mediate the process of reversing electric polarization of ferroelectrics. Formation energies of domain walls and energy barriers for moving domain walls indicate how difficult it is to form and grow a domain, respectively. We studied the atomistic structure of a few $180^{\circ}$ domain walls parallel to the $x$-axis in $\mathrm{As}_{2} \mathrm{X}_{3}$. For example, the structure of the $180^{\circ}$ domain wall in $\mathrm{As}_{2} \mathrm{~S}_{3}$ is shown schematically in the top panel of Fig. 4. Our calculations indicate the energy costs of forming and moving the $180^{\circ}$ domain wall along $x$-axis are reasonable compared to other ferroelectrics. In detail, the calculated domain-wall formation energies $E_{\text {form }}^{d w}$ are 89, 105, and $124 \mathrm{meV} /$ f.u. (i.e., 43, 50, and $58 \mathrm{meV} / \AA$ ) for monolayer $\mathrm{As}_{2} \mathrm{~S}_{3}$, $\mathrm{As}_{2} \mathrm{Se}_{3}$, and $\mathrm{As}_{2} \mathrm{Te}_{3}$, respectively. Previous cal- 
culations show the $E_{f o r m}^{d w}$ of group IV-VI materials range from $8 \mathrm{meV} / \AA$ to $116 \mathrm{meV} / \AA,{ }^{45}$ which covers those of $2 \mathrm{D} \mathrm{As}_{2} \mathrm{X}_{3}$ ferroelectrics. The $E_{\text {form }}^{d w}$ of $\operatorname{In}_{2} \mathrm{Se}_{3}$ is $220 \mathrm{meV} /$ f.u., ${ }^{25}$ comparable to those of $\mathrm{As}_{2} \mathrm{X}_{3}$. Assuming the thickness of monolayer $\mathrm{As}_{2} \mathrm{X}_{3}$ is $6.0 \AA$, we convert the $E_{\text {form }}^{d w}$ of $\mathrm{As}_{2} \mathrm{~S}_{3}, \mathrm{As}_{2} \mathrm{Se}_{3}$, and $\mathrm{As}_{2} \mathrm{Te}_{3}$ to be 115, 133 , and $155 \mathrm{~mJ} / \mathrm{m}^{2}$, which are in the same order as those of some ferroelectric oxides, such as $\mathrm{PbTiO}_{3}\left(132 \mathrm{~mJ} / \mathrm{m}^{2}\right.$ for $180^{\circ}$ domain wall and $35.2 \mathrm{~mJ} / \mathrm{m}^{2}$ for $90^{\circ}$ domain wall $)^{53}$ and $\mathrm{BiFeO}_{3}$ (205 to $\left.1811 \mathrm{~mJ} / \mathrm{m}^{2}\right),{ }^{54}$ but much higher than that of $\mathrm{BaTiO}_{3}\left(7.5 \mathrm{~mJ} / \mathrm{m}^{2}\right) .{ }^{53}$

Using nudged-elastic-band method, we calculate the energy barriers $E_{b a r r i e r}^{d w}$ for moving the $180^{\circ}$ domain walls are $233 \mathrm{meV} /$ f.u., $128 \mathrm{meV} /$ f.u., and $35 \mathrm{meV} /$ f.u. (i.e., 113 $\mathrm{meV} / \AA, 54 \mathrm{meV} / \AA$, and $16 \mathrm{meV} / \AA$ ) for monolayer $\mathrm{As}_{2} \mathrm{~S}_{3}, \mathrm{As}_{2} \mathrm{Se}_{3}$, and $\mathrm{As}_{2} \mathrm{Te}_{3}$, respectively, as shown in the bottom panel of Fig. 4. This suggests the $180^{\circ}$ domain wall of $\mathrm{As}_{2} \mathrm{X}_{3}$ becomes easier to shift as the chalcogen element $\mathrm{X}$ changes from sulfur to tellurium. Compared to bulk ferroelectrics, the $E_{b a r r i e r}^{d w}$ of monolayer $\mathrm{As}_{2} \mathrm{Te}_{3}$ and $\mathrm{As}_{2} \mathrm{Se}_{3}$ are of the same order-of-magnitude as those of bulk corundum derivatives ranging from $14 \mathrm{meV} /$ f.u. to 197 meV/f.u. ${ }^{55}$ Compared to other two-dimensional ferroelectrics, $E_{\text {barrier }}^{d w}$ of monolayer $\mathrm{As}_{2} \mathrm{~S}_{3}$ is more than an order-of-magnitude higher than those of group IV-VI two-dimensional ferroelectrics (less than $1.6 \mathrm{meV} / \AA$ ), ${ }^{45}$ but in similar order with that of monolayer $\mathrm{In}_{2} \mathrm{Se}_{3}$, which ranges from $280 \mathrm{meV} /$ f.u. to $400 \mathrm{meV} /$ f.u. ${ }^{25}$ These comparisons suggest that the energy costs for forming and moving the $180^{\circ}$ domain wall of $\mathrm{As}_{2} \mathrm{X}_{3}$ are reasonable.

Similar to monolayer group IV-VI compounds and black phosphorene, monolayer arsenic chalcogenides studied in this work are super flexible. We calculate Young's modulus of monolayer orpiment to be $8.6 \mathrm{~N} / \mathrm{m}$ along the $x$-axis and $33.6 \mathrm{~N} / \mathrm{m}$ along the $y$-axis, which is more than one-order-of-magnitude smaller than those of graphene $(345 \mathrm{~N} / \mathrm{m})^{56,57}$ and also significantly smaller than that of black phosphorene $(21 \sim 56 \mathrm{~N} / \mathrm{m}) .{ }^{58,59}$ To our knowledge, monolayer orpiment is among the softest 2D material ever fabricated. Such remarkable structural flexibility motivates us to investigate the piezoelectricity of arsenic chalcogenides.

We summarize the calculated elasticity tensor $C_{i j}$ and piezoelectric tensor elements $e_{i j}$ and $d_{i j}$ in Table 2. More details of calculating these tensor elements are presented in Supporting Information. Obviously, the piezoelectric strain coefficients $d_{i j}$ of $\mathrm{Pmn} 2_{1}$ and $\mathrm{Pc}$ phases are one-order-of-magnitude larger than those of common two-dimensional polar materials such as $2 \mathrm{H}-\mathrm{MoSe}_{2}\left(d_{11}=3.73 \mathrm{pm} / \mathrm{V}\right),{ }^{19} 2 \mathrm{H}$ $\mathrm{WSe}_{2}\left(d_{11}=2.79 \mathrm{pm} / \mathrm{V}\right),{ }^{19}$ hexagonal group III-V materials $\left(0.02<d_{11}<5.50 \mathrm{pm} / \mathrm{V}\right){ }^{18}$ and multilayer janus transition metal chalcogenide MoSTe $\left(5.7<d_{33}<13.5 \mathrm{pm} / \mathrm{V}\right){ }^{17}$ On the other hand, the piezoelectric stress constants $e_{i j}$ of arsenic chalcogenides are comparable with those of $2 \mathrm{H}-\mathrm{MoSe}_{2}, 2 \mathrm{H}-\mathrm{WSe}_{2}$, and so on. ${ }^{17-19}$ This indicates the large $d_{i j}$ coefficients of arsenic chalcogenides is originated from their superior flexibility, i.e., small elasticity tensor components. Compared to group IV-VI monolayers with giant piezoelectricity, the $d_{i j}$ coefficients of Pmn $2_{1}$ and Pc phases are on the same order as that of GeS, but two to four-times smaller than those of SnS, SnSe, and GeSe. ${ }^{20}$ The piezoelectric coefficients of $\mathrm{P} 2_{1}$ are much smaller than other phases. Interestingly, $\mathrm{P} 22_{1}$ $\mathrm{As}_{2} \mathrm{Se}_{3}$ shows weak negative piezoelectric effect along y-direction.

In summary, we employ ab initio methods to predict the intrinsic ferroelectricity and strong piezoelectricity in arsenic chalcogenides, which include the recently isolated monolayer orpiment. By analyzing the soft optical modes of the high-symmetry Pmmn structures of arsenic chalcogenides, we find these soft modes can lead to several undiscovered metastable polymorphs. The Pmn $2_{1}$ ferroelectric phases can be related to the soft $\mathrm{B}_{2 u}$ phonon mode of a highsymmetry Pmmn structure. We investigate the feasibility of switching the electrical polarization in the Pmn2 $2_{1}$ phase. The energy barrier of coherently flip all electrical dipoles and that of moving a $180^{\circ}$-domain wall in two-dimensional $\mathrm{Pmn} 2_{1} \mathrm{As}_{2} \mathrm{X}_{3}$ are in a proper range compared with other ferroelectrics. Moreover, superior structural flexibility results in large piezoelectric responses in a few polymorphs. Such a 
Table 2: Elasticity tensor elements $(\mathrm{N} / \mathrm{m})$ and piezoelectric coefficients $\left(10^{-10} \mathrm{C} / \mathrm{m}\right.$ for $e_{i j}$ and $\mathrm{pm} / \mathrm{V}$ for $\left.d_{i j}\right)$.

\begin{tabular}{ccccccccc}
\hline Space group (Point group) & Formula & $C_{11}$ & $C_{22}$ & $C_{12}$ & $e_{11}$ & $e_{12}$ & $d_{11}$ & $d_{12}$ \\
\hline \multirow{2}{*}{$\mathrm{Pmn}_{1}\left(\mathrm{C}_{2 v}\right)$} & $\mathrm{As}_{2} \mathrm{~S}_{3}$ & 11.07 & 43.38 & 10.40 & 4.36 & -1.75 & 55.7 & -17.4 \\
& $\mathrm{As}_{2} \mathrm{Se}_{3}$ & 13.86 & 41.76 & 10.03 & 6.71 & -1.49 & 61.7 & -18.4 \\
& $\mathrm{As}_{2} \mathrm{Te}_{3}$ & 18.09 & 34.65 & 9.72 & 9.09 & -1.48 & 61.9 & -21.6 \\
\hline & & $C_{11}$ & $C_{22}$ & $C_{12}$ & $e_{21}$ & $e_{22}$ & $d_{21}$ & $d_{22}$ \\
\hline \multirow{2}{*}{$\mathrm{P} 2_{1}\left(\mathrm{C}_{2}\right)$} & $\mathrm{As}_{2} \mathrm{~S}_{3}$ & 18.63 & 16.29 & 3.42 & -0.85 & 0.22 & -5.0 & 2.4 \\
& $\mathrm{As}_{2} \mathrm{Se}_{3}$ & 21.51 & 23.76 & 2.65 & -0.91 & -0.33 & -4.1 & -0.9 \\
\hline $\mathrm{Pc}\left(\mathrm{C}_{s}\right)$ & $\mathrm{As}_{2} \mathrm{~S}_{3}$ & 21.63 & 9.25 & 8.00 & -2.77 & 2.69 & -34.7 & 59.1 \\
\hline
\end{tabular}

unique combination of unusual structures, pliability, strong piezoelectricity, and predicted ferroelectricity make monolayer arsenic chalcogenides new platforms of studying polar materials. They are also convincing candidates for small-sized, flexible electronic devices.

Computation Details: Our first-principles calculations are based on pseudopotential density functional theory implemented in Quantum Espresso $^{60,61}$ and PARSEC. ${ }^{62,63}$ More technical details are presented in Supporting Information, which cites these references. ${ }^{37-39,64-68}$

\section{Acknowledgement}

W.G. and J.R.C. acknowledge support from a subaward from the Center for Computational Study of Excited-State Phenomena in Energy Materials at the Lawrence Berkeley National Laboratory, which is funded by the U.S. Department of Energy, Office of Science, Basic Energy Sciences, Materials Sciences and Engineering Division under Contract No. DEAC0205CH11231, as part of the Computational Materials Sciences Program. Computational resources are provided by the Texas Advanced Computing Center (TACC).

\section{Supporting Information Avail- able}

The supporting information presents more details on piezoelectric tensors, phonon spectra, molecular dynamics simulation, transition path for reversing electric polarization, and structure parameters of arsenic chalcogenides polymorphs. This material is available free of charge via the internet at http://pubs.acs.org.

\section{References}

(1) Li, W.; Ji, L.-J. Perovskite ferroelectrics go metal free. Science 2018, 361, 132-132.

(2) Scott, J. F.; Paz de Araujo, C. A. Ferroelectric Memories. Science 1989, 246, 1400-1405.

(3) Fang, L.; You, L.; Liu, J.-M. Ferroelectric Materials for Energy Applications; John Wiley \& Sons, Ltd, 2018; Chapter 9, pp 265-309.

(4) Setter, N.; Damjanovic, D.; Eng, L.; Fox, G.; Gevorgian, S.; Hong, S.; Kingon, A.; Kohlstedt, H.; Park, N. Y.; Stephenson, G. B.; Stolitchnov, I.; Taganstev, A. K.; Taylor, D. V.; Yamada, T.; Streiffer, S. Ferroelectric thin films: Review of materials, properties, and applications. Journal of Applied Physics 2006, 100, 051606.

(5) Park, M. H.; Lee, Y. H.; Kim, H. J.; Kim, Y. J.; Moon, T.; Kim, K. D.; Müller, J.; Kersch, A.; Schroeder, U.; Mikolajick, T.; Hwang, C. S. Ferroelectricity and Antiferroelectricity of Doped 
Thin $\mathrm{HfO}_{2}$-Based Films. Advanced $\mathrm{Ma}$ terials 2015, 27, 1811-1831.

(6) Böscke, T. S.; Müller, J.; Bräuhaus, D.; Schröder, U.; Böttger, U. Ferroelectricity in hafnium oxide thin films. Applied Physics Letters 2011, 99.

(7) Junquera, J.; Ghosez, P. Critical thickness for ferroelectricity in perovskite ultrathin films. Nature 2003, 422, 506-509.

(8) Mehta, R. R.; Silverman, B. D.; Jacobs, J. T. Depolarization fields in thin ferroelectric films. Journal of Applied Physics 1973, 44, 3379-3385.

(9) Wurfel, P.; Batra, I. P.; Jacobs, J. T. Polarization Instability in Thin Ferroelectric Films. Phys. Rev. Lett. 1973, 30, 12181221.

(10) Fong, D. D.; Stephenson, G. B.; Streiffer, S. K.; Eastman, J. A.; Auciello, O.; Fuoss, P. H.; Thompson, C. Ferroelectricity in Ultrathin Perovskite Films. Science 2004, 304, 1650-1653.

(11) Lee, S. R.; Baasandorj, L.; Chang, J. W.; Hwang, I. W.; Kim, J. R.; Kim, J.-G.; Ko, K.-T.; Shim, S. B.; Choi, M. W.; You, M.; Yang, C.-H.; Kim, J.; Song, J. First Observation of Ferroelectricity in $1 \mathrm{~nm}$ Ultrathin Semiconducting BaTiO3 Films. Nano Letters 2019, 19, 2243-2250.

(12) Fei, Z.; Zhao, W.; Palomaki, T. A.; Sun, B.; Miller, M. K.; Zhao, Z.; Yan, J.; $\mathrm{Xu}$, X.; Cobden, D. H. Ferroelectric switching of a two-dimensional metal. $\mathrm{Na}$ ture 2018, 560, 336-339.

(13) Liu, F. et al. Room-temperature ferroelectricity in CuInP $2 \mathrm{~S} 6$ ultrathin flakes. $\mathrm{Na}-$ ture Communications 2016, 7 .

(14) Chang, K.; Liu, J.; Lin, H.; Wang, N.; Zhao, K.; Zhang, A.; Jin, F.; Zhong, Y.; $\mathrm{Hu}$, X.; Duan, W.; Zhang, Q.; Fu, L.; Xue, Q. K.; Chen, X.; Ji, S. H. Discovery of robust in-plane ferroelectricity in atomic-thick SnTe. Science 2016, 353, 274-278.
(15) Zhou, Y.; Wu, D.; Zhu, Y.; Cho, Y.; He, Q.; Yang, X.; Herrera, K.; Chu, Z.; Han, Y.; Downer, M. C.; Peng, H.; Lai, K. Out-of-Plane Piezoelectricity and Ferroelectricity in Layered $\alpha-\mathrm{In}_{2} \mathrm{Se}_{3}$ Nanoflakes. Nano Letters 2017, 17, 5508-5513.

(16) Sharma, P.; Xiang, F.-X.; Shao, D.F.; Zhang, D.; Tsymbal, E. Y.; Hamilton, A. R.; Seidel, J. A room-temperature ferroelectric semimetal. Science Advances 2019, 5 .

(17) Dong, L.; Lou, J.; Shenoy, V. B. Large In-Plane and Vertical Piezoelectricity in Janus Transition Metal Dichalchogenides. ACS Nano 2017, 11, 8242-8248.

(18) Blonsky, M. N.; Zhuang, H. L.; Singh, A. K.; Hennig, R. G. Ab Initio Prediction of Piezoelectricity in Two-Dimensional Materials. ACS Nano 2015, 9, 9885-9891.

(19) Duerloo, K.-A. N.; Ong, M. T.; Reed, E. J. Intrinsic Piezoelectricity in Two-Dimensional Materials. The Journal of Physical Chemistry Letters 2012, 3, 2871-2876.

(20) Fei, R.; Kang, W.; Yang, L. Ferroelectricity and Phase Transitions in Monolayer Group-IV Monochalcogenides. Phys. Rev. Lett. 2016, 117, 097601.

(21) Cui, C.; Xue, F.; Hu, W. J.; Li, L. J. Twodimensional materials with piezoelectric and ferroelectric functionalities. $n p j$ 2D Materials and Applications 2018, 2.

(22) Wu, M.; Zeng, X. C. Intrinsic Ferroelasticity and/or Multiferroicity in TwoDimensional Phosphorene and Phosphorene Analogues. Nano Letters 2016, 16, 3236-3241, PMID: 27096689.

(23) Mehboudi, M.; Dorio, A. M.; Zhu, W.; van der Zande, A.; Churchill, H. O. H.; Pacheco-Sanjuan, A. A.; Harriss, E. O.; 
Kumar, P.; Barraza-Lopez, S. TwoDimensional Disorder in Black Phosphorus and Monochalcogenide Monolayers. Nano Letters 2016, 16, 1704-1712, PMID: 26866878.

(24) Gao, Y.; Wu, M.; Zeng, X. C. Phase transitions and ferroelasticity-multiferroicity in bulk and two-dimensional silver and copper monohalides. Nanoscale Horiz. 2019, 4, 1106-1112.

(25) Ding, W.; Zhu, J.; Wang, Z.; Gao, Y.; Xiao, D.; Gu, Y.; Zhang, Z.; Zhu, W. Prediction of intrinsic two-dimensional ferroelectrics in In 2 Se 3 and other III 2 -VI 3 van der Waals materials. Nature Communications 2017, 8 .

(26) Chandrasekaran, A.; Mishra, A.; Singh, A. K. Ferroelectricity, Antiferroelectricity, and Ultrathin 2D Electron/Hole Gas in Multifunctional Monolayer MXene. Nano Letters 2017, 17, 3290-3296.

(27) Lin, L.-F.; Zhang, Y.; Moreo, A.; Dagotto, E.; Dong, S. Frustrated Dipole Order Induces Noncollinear Proper Ferrielectricity in Two Dimensions. Phys. Rev. Lett. 2019, 123, 067601.

(28) Higashitarumizu, N.; Kawamoto, H.; Lee, C.-J.; Lin, B.-H.; Chu, F.-H.; Yonemori, I.; Nishimura, T.; Wakabayashi, K.; Chang, W.-H.; Nagashio, K. Purely in-plane ferroelectricity in monolayer $\mathrm{SnS}$ at room temperature. Nature Communications 2020, 11, 2428.

(29) Chang, K.; Küster, F.; Miller, B. J.; Ji, J.-R.; Zhang, J.-L.; Sessi, P.; BarrazaLopez, S.; Parkin, S. S. P. Microscopic Manipulation of Ferroelectric Domains in SnSe Monolayers at Room Temperature. Nano Letters 2020, 20, 6590-6597, PMID: 32809837.

(30) Šiškins, M.; Lee, M.; Alijani, F.; van Blankenstein, M. R.; Davidovikj, D.; van der Zant, H. S. J.; Steeneken, P. G.
Highly Anisotropic Mechanical and Optical Properties of 2D Layered As2S3 Membranes. ACS Nano 2019, 13, 1084510851.

(31) Momma, K.; Izumi, F. VESTA3 for threedimensional visualization of crystal, volumetric and morphology data. Journal of Applied Crystallography 2011, 44, 12721276.

(32) Kampf, A.; Downs, R.; Housley, R.; Jenkins, R.; Hyrsl, J. Anorpiment, As2S3, the triclinic dimorph of orpiment. Mineralogical Magazine 2011, 75, 2857-2867.

(33) Perdew, J. P.; Burke, K.; Ernzerhof, M. Generalized Gradient Approximation Made Simple. Phys. Rev. Lett. 1996, 7ry, 3865-3868.

(34) Stergiou, A. C.; Rentzeperis, P. J. The crystal structure of arsenic selenide, As2Se3. Zeitschrift für Kristallographie Crystalline Materials 1985, 173, 185-191.

(35) Resta, R. Macroscopic polarization in crystalline dielectrics: the geometric phase approach. Rev. Mod. Phys. 1994, 66, 899-915.

(36) King-Smith, R. D.; Vanderbilt, D. Theory of polarization of crystalline solids. Phys. Rev. B 1993, 47, 1651-1654.

(37) Mullen, D.; Nowacki, W. Refinement of the crystal structures of realgar, AsS and orpiment, As2S3. Zeitschrift für Kristallographie - Crystalline Materials 1972, 136, 48.

(38) Baroni, S.; de Gironcoli, S.; Dal Corso, A.; Giannozzi, P. Phonons and related crystal properties from density-functional perturbation theory. Rev. Mod. Phys. 2001, 73, 515-562.

(39) Berendsen, H. J. C.; Postma, J. P. M.; van Gunsteren, W. F.; DiNola, A.; Haak, J. R. Molecular dynamics with coupling to an external bath. The Journal of Chemical Physics 1984, 81, 3684-3690. 
(40) Henkelman, G.; Jónsson, H. Improved tangent estimate in the nudged elastic band method for finding minimum energy paths and saddle points. The Journal of Chemical Physics 2000, 113, 9978-9985.

(41) Barraza-Lopez, S.; Kaloni, T. P.; Poudel, S. P.; Kumar, P. Tuning the ferroelectric-to-paraelectric transition temperature and dipole orientation of group-IV monochalcogenide monolayers. Phys. Rev. B 2018, 97, 024110.

(42) Yuan, S.; Luo, X.; Chan, H. L.; Xiao, C.; Dai, Y.; Xie, M.; Hao, J. Roomtemperature ferroelectricity in MoTe2 down to the atomic monolayer limit. $\mathrm{Na}$ ture Communications 2019, 10, 1775.

(43) Huan, T. D.; Sharma, V.; Rossetti, G. A.; Ramprasad, R. Pathways towards ferroelectricity in hafnia. Phys. Rev. B 2014, 90, 064111.

(44) Sang, X.; Grimley, E. D.; Schenk, T.; Schroeder, U.; LeBeau, J. M. On the structural origins of ferroelectricity in HfO2 thin films. Applied Physics Letters 2015, 106, 162905.

(45) Wang, H.; Qian, X. Two-dimensional multiferroics in monolayer group IV monochalcogenides. 2D Materials 2017, 4, 015042 .

(46) Cui, C. et al. Intercorrelated In-Plane and Out-of-Plane Ferroelectricity in Ultrathin Two-Dimensional Layered Semiconductor In2Se3. Nano Letters 2018, 18, 12531258 .

(47) Beckman, S. P.; Wang, X.; Rabe, K. M.; Vanderbilt, D. Ideal barriers to polarization reversal and domain-wall motion in strained ferroelectric thin films. Phys. Rev. B 2009, 79, 144124.

(48) Ye, M.; Vanderbilt, D. Ferroelectricity in corundum derivatives. Phys. Rev. B 2016, 93, 134303.
(49) Brehm, J. A.; Neumayer, S. M.; Tao, L.; O'Hara, A.; Chyasnavichus, M.; Susner, M. A.; McGuire, M. A.; Kalinin, S. V.; Jesse, S.; Ganesh, P.; Pantelides, S. T.; Maksymovych, P.; Balke, N. Tunable quadruple-well ferroelectric van der Waals crystals. Nature Materials 2020, 19, 43-48.

(50) Ai, H.; Song, X.; Qi, S.; Li, W.; Zhao, M. Intrinsic multiferroicity in two-dimensional VOCl2 monolayers. Nanoscale 2019, 11, 1103-1110.

(51) Xu, B.; Xiang, H.; Xia, Y.; Jiang, K.; Wan, X.; He, J.; Yin, J.; Liu, Z. Monolayer AgBiP2Se6: an atomically thin ferroelectric semiconductor with out-plane polarization. Nanoscale 2017, 9, 8427-8434.

(52) Jia, Y.; Zhao, M.; Gou, G.; Zeng, X. C.; Li, J. Niobium oxide dihalides NbOX2: a new family of two-dimensional van der Waals layered materials with intrinsic ferroelectricity and antiferroelectricity. Nanoscale Horiz. 2019, 4, 1113-1123.

(53) Meyer, B.; Vanderbilt, D. Ab initio study of ferroelectric domain walls in $\mathrm{PbTiO}_{3}$. Phys. Rev. B 2002, 65, 104111.

(54) Lubk, A.; Gemming, S.; Spaldin, N. A. First-principles study of ferroelectric domain walls in multiferroic bismuth ferrite. Phys. Rev. B 2009, 80, 104110.

(55) Ye, M.; Vanderbilt, D. Domain walls and ferroelectric reversal in corundum derivatives. Phys. Rev. B 2017, 95, 014105.

(56) Kudin, K. N.; Scuseria, G. E.; Yakobson, B. I. $\mathrm{C}_{2} \mathrm{~F}, \mathrm{BN}$, and $\mathrm{C}$ nanoshell elasticity from ab initio computations. Phys. Rev. B 2001, 64, 235406.

(57) Akinwande, D. et al. A review on mechanics and mechanical properties of 2D materials - Graphene and beyond. Extreme Mechanics Letters 2017, 13, 42 - 77.

(58) Jiang, J.-W.; Park, H. S. Mechanical properties of single-layer black phosphorus. 
Journal of Physics D: Applied Physics 2014, 47, 385304.

(59) Wei, Q.; Peng, X. Superior mechanical flexibility of phosphorene and few-layer black phosphorus. Applied Physics Letters 2014, 104, 251915.

(60) Giannozzi, P. et al. Advanced capabilities for materials modelling with QUANTUM ESPRESSO. Journal of Physics: Condensed Matter 2017, 29, 465901.

(61) Giannozzi, P. et al. QUANTUM ESPRESSO: a modular and opensource software project for quantum simulations of materials. Journal of Physics: Condensed Matter 2009, 21, 395502.

(62) Chelikowsky, J. R.; Troullier, N.; Saad, Y. Finite-difference-pseudopotential method: Electronic structure calculations without a basis. Physical Review Letters 1994, 72, 1240-1243.

(63) Kronik, L.; Makmal, A.; Tiago, M. L.; Alemany, M. M. G.; Jain, M.; Huang, X.; Saad, Y.; Chelikowsky, J. R. PARSEC - the pseudopotential algorithm for realspace electronic structure calculations: recent advances and novel applications to nano-structures. physica status solidi (b) 2006, 243, 1063-1079.

(64) Perdew, J. P.; Ruzsinszky, A.; Csonka, G. I.; Vydrov, O. A.; Scuseria, G. E.; Constantin, L. A.; Zhou, X.; Burke, K. Restoring the Density-Gradient Expansion for Exchange in Solids and Surfaces. Phys. Rev. Lett. 2008, 100, 136406.

(65) Corso, A. D. Pseudopotentials periodic table: From $\mathrm{H}$ to $\mathrm{Pu}$. Computational Materials Science 2014, 95, 337 - 350.

(66) Vanderbilt, D. Soft self-consistent pseudopotentials in a generalized eigenvalue formalism. Phys. Rev. B 1990, 41, 78927895 .
(67) Troullier, N.; Martins, J. L. Efficient pseudopotentials for plane-wave calculations. Phys. Rev. B 1991, 43, 1993-2006.

(68) Nye, J. F. Physical properties of crystals: their representation by tensors and matrices; Clarendon Press: Oxford, UK, 1985. 
Graphical TOC Entry

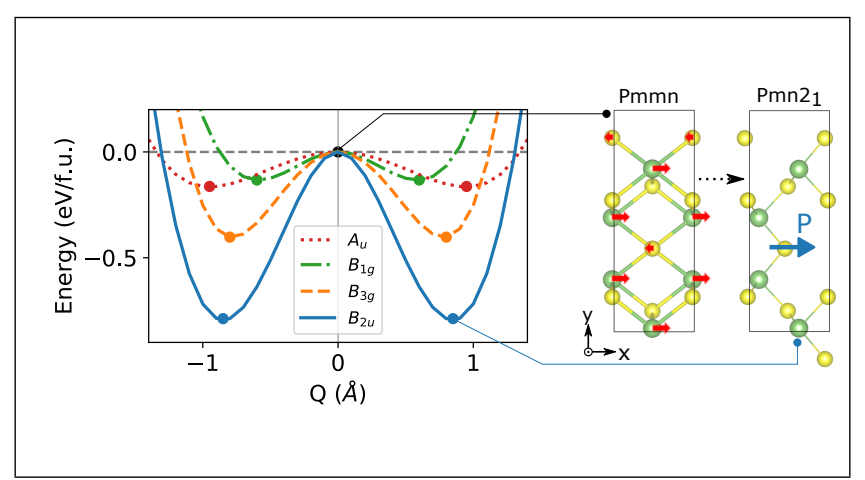

